Ciência Florestal, Santa Maria, v. 22, n. 3, p. 433-443, jul.-set., 2012

ISSN 0103-9954

\title{
EFEITO COMPETICIONAL EM TESTES DE PROGÊNIES DE EUCALIPTO
}

\author{
EVALUATION OF COMPETITION EFFECT IN Eucalyptus PROGENY TESTS
}

\author{
Bruno Ettore Pavan ${ }^{1}$ Rinaldo César de Paula ${ }^{2}$ Dilermando Perecin ${ }^{3}$ \\ Liliam Silvia Candido ${ }^{4}$ Edimar Aparecido Scarpinati ${ }^{5}$
}

\begin{abstract}
RESUMO
Muitos estudos têm recomendado o uso de parcelas pequenas na experimentação florestal, porém, não consideram o aumento da competição intergenotípica que isto acarreta e que pode resultar na seleção incorreta dos materiais genéticos. O objetivo deste trabalho foi o de avaliar sete covariáveis de competição sobre o caráter volume de madeira em dois testes de progênies de Eucalyptus spp. O delineamento utilizado foi de blocos casualizados, com seis repetições de 10 plantas. As sete covariáveis analisadas foram índice de competição de Hegyi (IC), autocompetição (AT), alocompetição (AL), média da autocompetição (MAT), média da alocompetição (MAL) e média aritmética dos quatro (M4) e oito vizinhos mais próximos (M8). Essas covariáveis foram avaliadas individualmente bem com em todas as suas possíveis combinações, obtendo-se estimativas de componentes de variâncias e suas alterações com o emprego das mesmas. A competição influenciou os resultados de análises de testes de progênies de eucalipto, em que, autocompetição e alocompetição interferem de forma diferenciada. As covariáveis mais influentes são a MAT, a MAL e o IC. A rotina de análise que apresentou melhores resultados foi a que incluiu as covariáveis IC/MAT, sendo eficiente na redução do efeito competicional em testes de progênies de eucalipto.
\end{abstract}

Palavras-chave: Eucalyptus spp.; competição intergenotípica; famílias de polinização aberta.

\section{ABSTRACT}

Many studies have recommended the use of small plots for forest experiments, although they do not consider the inter-genotype competition increase. If this competition is not isolated from the mathematics model, it can lead to incorrect selection of genetic materials. The aim of this work was to evaluate the effect of seven competition covariates in two Eucalyptus spp. progeny tests. Data from the two half-sib eucalyptus progenies were analyzed, using the randomized blocks design. The seven analyzed covariates were Hegyl's competition index (IC), self-competition (AT), alo competition (AL), self-competition mean (MAT), alo competition mean (MAL), and arithmetic means of four (M4) and eight (M8) nearest neighbors. Individual and combined analyses of covariates were used for the wood volume trait. All the variance components and the changes caused by covariates use were evaluated. The competition affects the results of eucalypt progeny analysis in different ways, according to its type, self or alo competition. Most influential covariates were MAT, MAL and IC. Most promising results of competition effects reduction were observed for the IC/ MAT covariates inclusion in eucalypt progeny tests.

Keywords: Eucalyptus spp.; inter-genotype competition; half-sib families.

1. Engenheiro Agrônomo, Dr., Professor Adjunto do Departamento de Engenharias da Universidade Federal do Piauí, Rod. BR 135, km 3, CEP 64900-000, Bom Jesus (PI). pavan@ufpi.edu.br

2. Engenheiro Florestal, Dr., Professor Adjunto do Departamento de Produção Vegetal, Faculdade de Ciências Agrárias e Veterinárias, Rod. Paulo Donato Castellani, km 5, CEP 14884-900, Jaboticabal (SP). Bolsista PQ-CNPq. rcpaula@fcav.unesp.br

3. Engenheiro Agrônomo, Dr., Professor Titular do Departamento de Ciências Exatas, Faculdade de Ciências Agrárias e Veterinárias, Rod. Paulo Donato Castellani, km 5, CEP 14884-900, Jaboticabal (SP). Bolsista PQ-CNPq. perecin@fcav.unesp.br

4. Bióloga, Dra ${ }^{\mathrm{a}}$., Pós-Doutoranda do Laboratório de Melhoramento Genético Vegetal, Universidade Estadual Norte Fluminense Darcy Ribeiro, Campos dos Goytacazes, Av. Alberto Lamego 2000, Horto28013-602, CEP 21000-000, Goytacazes (RJ). lcandido@uenf.com.br

5. Administrador, Msc., Pesquisador de Melhoramento Florestal da Empresa Fibria. Rodovia MS 395, km 20, Zona rural, Caixa Postal 529, CEP 79601-970, Três Lagoas (MS). edimar.scarpinati@fibria.com.br

Recebido para publicação em 05/10/2009 e aceito em 19/07/2011

Ci. Fl., v. 22, n. 3, jul.-set., 2012 


\section{INTRODUÇÃO}

A separação da variação genética da não genética é um dos principais objetivos da genética quantitativa (FALCONER, 1987), onde o sucesso de programas de melhoramento de plantas depende de uma ampla gama de fatores, tanto técnicos como operacionais. Dentre esses fatores, o melhoramento genético depende quase que totalmente da adoção de métodos acurados de seleção (RESENDE, 2000), que identifiquem corretamente as variáveis envolvidas nesse processo de seleção.

Muitos estudos têm recomendado o uso de parcelas pequenas na experimentação florestal, porém, não consideram o aumento da competição intergenotípica que isto acarreta. Tratando de espécies ou materiais genéticos diferentes, a competição pode resultar na diminuição da produção, comprometendo o rendimento econômico ou, no caso de experimentos de melhoramento, resultar na escolha de indivíduos que não sejam superiores, mas que possuam poder de agressividade competicional maior (LEONARDECZ NETO et al., 2003).

Entretanto, a maioria dos experimentos florestais de seleção não leva em conta as variáveis de competição, causando, desta forma, um viés que não condiz com o evento biológico, o que pode acarretar em uma seleção incorreta. Mesmo quando incluída no modelo estatístico, geralmente se adota uma única variável de competição, a mais comumente utilizada é o Índice de Hegyi (1974), como nos trabalhos de Leonardecz Neto et al. (2003) e Lins (2004).

Existem diversas formas de competição entre as árvores, como a competição intergenotípica e intragenotípica e suas variantes, todavia estes efeitos de competição são pouco estudados e a forma como eles interferem em testes de progênies de eucalipto é pouco conhecida. Quando consideradas nas análises, assume-se que o crescimento dos indivíduos da parcela está correlacionado aos seus vizinhos, devido ao microambiente comum ou pelo gradiente de recursos (água, luz e nutrientes) criado pelo desenvolvimento destes (LEONARDECZ NETO et al., 2003).

Nos modelos utilizados para análise de testes de progênies, com objetivo de seleção, não é comum considerar os efeitos de competição, por não haver rotinas estabelecidas e a definição de variáveis para se corrigir esse efeito. Deste modo, é necessário o estabelecimento de rotinas de análise e a escolha de covariáveis de correção que melhor representem testes genéticos. Desta forma, procurase minimizar, o máximo possível, a competição entre árvores vizinhas, como no caso de testes de progênies de eucalipto.

Sendo o efeito competicional uma importante fonte de variação experimental, este trabalho teve como objetivo a avaliação do efeito de competição por sete covariáveis e suas combinações, sobre o caráter volume de madeira em dois testes de progênies de polinização aberta de eucalipto.

\section{MATERIAL E MÉTODOS}

\section{Caracterização dos experimentos}

Foram usados dados de dois testes de progênies de polinização aberta de eucalipto, pertencentes à Votorantim Celulose e Papel, instalados no município de Guatapará, São Paulo, a $21^{\circ} 29^{\prime} 0,73$ " de latitude sul e $47^{\circ} 58^{\prime} 59,84^{\prime \prime}$ de longitude oeste. O experimento 1 (EXP1) foi implantado no ano de 1996 e o experimento 2 (EXP2) em 1999. Foram avaliados o crescimento em altura (ALT) e a circunferência a altura do peito (CAP), ambos aos sete anos de idade. Destas medições foi determinado o volume cilíndrico das árvores, aplicando-se o fator de forma utilizado pela empresa, para classe diamétrica, para a obtenção do volume comercial com casca.

$\mathrm{O}$ delineamento experimental foi o de blocos casualizados com seis repetições em ambos os experimentos, sendo o EXP1 composto por quatro testemunhas (clones de Eucalyptus grandis $x$ Eucalyptus urophylla) e 49 tratamentos (famílias de polinização aberta de Eucalyptus grandis) e o EXP2 por 44 tratamentos (famílias de polinização aberta de Eucalyptus grandis $x$ Eucalyptus urophylla). As parcelas obedeceram a uma disposição linear, contendo 10 plantas, totalizando 3.180 plantas no EXP1 e 2.640 no EXP2, no espaçamento de 3,0 x $2,5 \mathrm{~m}$, formando assim um teste de progênies de eucalipto de 2,385 ha no EXP1, e outro de 1,98 ha no EXP2.

\section{Análise do efeito competicional}

Para a análise de competição foram usadas sete covariáveis calculadas a partir do desempenho de suas árvores vizinhas, conforme Figura 1, sendo as covariáveis testadas a seguir definidas.

\section{Índice de competição (IC) de Hegyi (1974)}

Este índice consiste na distância ponderada agregada dos raios de competição pela característica 


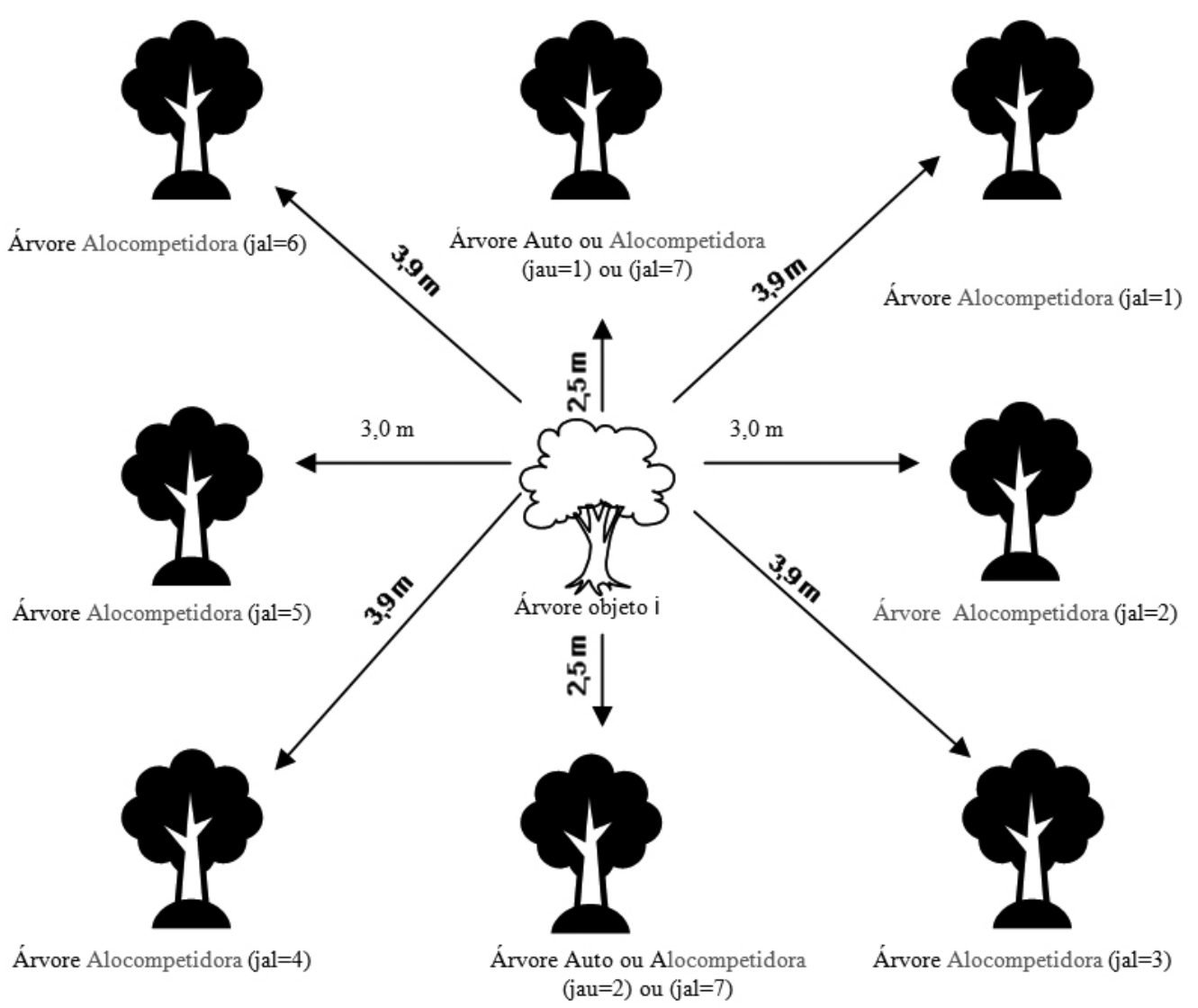

FIGURA 1: Disposição das árvores competidoras para cálculo das covariáveis.

FIGURE 1: Disposition of the trees competing for the calculation of covariates.

silvicultural das árvores consideradas como competidoras em relação à árvore objeto.

$$
I C_{i}=\sum_{j=1}^{n}\left(D_{j} / D_{i}\right) D_{i s t_{i j}}
$$

Em que:

$I C_{i}=$ índice de competição da arvore $i$;

$D_{j} / D_{i}=$ volume do competidor $j$ expresso em relação ao volume da árvore objeto $i$;

Dist $_{i j}=$ Distância entre o competidor $j$ e a árvore objeto $i$;

$n=$ número de árvores competidoras.

No emprego desta equação foram consideradas as oito árvores vizinhas mais próximas (competidoras).

\section{Autocompetição (AT)}

Falha dentro da parcela, ocasionando menor ou maior grau de competição entre a árvore objeto e as árvores vizinhas do mesmo material genético, podendo variar de 0 a 0,25 (de nenhuma a duas árvores; será zero no caso de falha das duas árvores vizinhas), considerando que as oito árvores vizinhas tenham um valor de competição igual a 1. Esta competição ocorre dentro da parcela.

\section{Média da autocompetição (MAT)}

Média aritmética da característica silvicultural das árvores autocompetidoras. Sendo expressa por:

$$
M A T=\sum_{j=1}^{n} j_{a u} / n
$$

Sendo: $j_{a u}=$ volume da árvore autocompetidora;

$$
n=\text { número de árvores autocompetidoras. }
$$

\section{Alocompetição (AL)}

Falha nas parcelas adjacentes, ocasionando menor ou maior grau de competição entre a árvore objeto e as árvores vizinhas de material genético diferente, podendo variar de 0 a 0,875 (de nenhuma a sete árvores; será zero no caso de falhas nas árvores vizinhas), considerando que as oito árvores 
vizinhas tenham um valor de competição igual a 1. Esta competição ocorre entre a árvore objeto e árvores de parcelas adjacentes.

\section{Média da Alocompetição (MAL)}

Média aritmética das características silviculturais das árvores alocompetidoras;

$$
M A T=\sum_{j=1}^{n} j_{a l} / n
$$

Em que: $j_{a l}=$ volume das árvores alocompetidoras;

$n=$ número de árvores alocompetidoras.

\section{Média 4 e 8 (M4, M8)}

Para a determinação da média 4 e 8 , foram consideradas as quatro (Média $4-\mathrm{M} 4$ ) e as oito (Média 8 - M8) árvores vizinhas mais próximas, sendo determinada a média aritmética do volume das árvores competidoras em questão à árvore objeto.

Efetuou-se o teste de significância do coeficiente de regressão linear simples $(b)$, de cada covariável e se ela se ajustava a uma única regressão linear, forma ideal, ou a nenhuma, ou a várias regressões lineares, formas indesejadas, por covariável e tratamento.

$$
y=a+b x
$$

Em que:

$y=$ a variável volume de madeira;

$a=$ constante da regressão linear simples;

$b=$ coeficiente de regressão linear simples;

$x=$ valor da covariável aplicada.

As covariáveis que não foram significativas, ou apresentaram alta significância para interação covariável x tratamento, foram descartadas do estudo. As covariáveis foram testadas individualmente e em todas as suas combinações, duas a duas até seis a seis. Duas covariáveis não foram testadas conjuntamente por serem sobrepostas (média 4 e 8).

\section{Estimativas de componentes de variâncias e parâmetros genéticos}

A análise de variância foi realizada com base nos dados de plantas individuais sobreviventes do teste de progênies para o caráter volume. Para escolha do valor representativo de cada progênie, os valores genotípicos do caráter estudado foram obtidos pelo método de Máxima Verossimilhança Restrita - REML, aplicado ao modelo linear misto.
Atualmente, a metodologia de modelos mistos tem se tornado mais acessível aos usuários graças à sua implementação em sistemas estatístico computacionais de ampla divulgação, como o SAS (Statistical Analysis System) (DUARTE e VENCOVSKY, 2001). No melhoramento de plantas tem sido utilizada, principalmente em análises de espécies florestais e perenes, a exemplo de Garcia e Nogueira (2005), Rocha et al. (2007), Costa et al. (2002) e Simeão et al. (2002).

\section{Ajuste pela covariável de competição e análise de covariância}

O modelo linear para descrição do valor individual, comumente utilizado em um delineamento em blocos casualizados, indica que uma dada variável resposta é função dos efeitos de ambiente comum (bloco), do potencial genético (família), do indivíduo na parcela (desvio do fenótipo dentro) e de um resíduo dado pela variação que é decorrente de efeitos não mensurados, ou que não são isolados no delineamento (no caso dos blocos aumentados, o resíduo é estimado pela variação dos clones dentro de parcela). Os fatores não incluídos no modelo matemático, e obviamente nas análises, geram inadequação do modelo. Não sendo o efeito do fator isolado do resíduo, ele poderá levar a um viés nas estimativas dos parâmetros (LEONARDECZ NETO et al., 2003).

Um dos métodos utilizados para ajustar a variável resposta, quando influenciada por uma variável adicional, é a análise de covariância. Este método permite corrigir os dados, de forma que, as variações devidas à covariável sejam minimizadas, ou até mesmo anuladas, deste modo, fornecendo estimativas corretas dos componentes de variância e de outros parâmetros.

Considerando um modelo estatístico tradicional de delineamento em blocos casualizados e incluindo uma covariável $\mathrm{X}$, tem-se:

$$
Y_{i j k}=m+f_{i}+b_{j}+\beta\left(X_{i j k}\right)+e_{i j}+d_{i j k}
$$

Em que:

$Y_{i j k}=$ valor do $k$-ésimo indivíduo no $j$-ésimo bloco da $i$-ésima progênie;

$m=$ média geral;

$f_{i}=$ efeito aleatório da progênie $i(i=1,2$, $3, \ldots, I)$;

$b_{j}=$ efeito fixo do bloco $j(j=1,2,3, \ldots, J)$;

$e_{i j}=$ erro experimental, inerente à parcela $i j$;

$d_{i j k}=$ desvio fenotípico do indivíduo $k$ dentro da parcela $i j(k=1,2,3, \ldots, K)$. 
$\beta=$ coeficiente de regressão linear entre a variável resposta e a covariável;

$X_{i j k}=$ valor da variável, no caso, de competição associada ao indivíduo $i j k$.

Considerando que $\mathrm{X}$ pode assumir qualquer uma das covariáveis de competição testadas no trabalho, de forma isolada ou conjunta, o modelo estatístico pode ser corrigido com mais de uma covariável.

Assim:

$Y_{i j k}=m+f_{i}+b_{j}+\beta_{1}\left(X_{1 i j k}\right) \ldots+\beta_{n}\left(X_{n i j k}\right)+e_{i j}+d_{i j k}$

As pressuposições desejadas para se proceder à análise, incluindo uma covariável, conforme Ramalho et al. (2000), são as seguintes:

i. A covariável é uma variável quantitativa, medida sem erro e independente dos tratamentos;

ii. Aregressão entre a variável Y e a covariável $\mathrm{C}$, após a remoção das diferenças de blocos e tratamentos, é linear e independente de blocos e tratamentos;

iii. $\mathrm{O}$ resíduo é normalmente e independentemente distribuído, com média zero e variância $\sigma_{e}^{2}$.

Para as covariáveis IC, MAT, MAL, M4 e M8, estas três pressuposições não são satisfeitas na íntegra, uma vez que as estimativas destas covariáveis de competição de cada indivíduo violam a primeira condição. Mas, trabalhos como o de Storck e Vencovsky (1994) demonstram que a utilização de índices contendo erros de estimativas e dependentes dos dados, não deve ser a única ou a principal preocupação para se fazer uma análise confiável. A escolha adequada do local para a realização dos experimentos, a redução da variância do erro experimental e de blocos são tão ou mais importantes do que o erro associado à covariável. Esses autores também verificaram que, com número adequado de tratamentos e/ou blocos, a dependência da covariável X e a variável resposta $\mathrm{Y}$, bem como os erros contidos em $\mathrm{X}$, provocam prejuízos mínimos à análise de covariância. Para atenuar esses efeitos, no presente trabalho, foi feita uma correção dos dados retirando-se dados discrepantes da análise (dados das árvores que apresentaram volume inferior a $0,015 \mathrm{~m}^{3}$ ), tornando assim a regressão mais uniforme para todos os tratamentos.

$\mathrm{Na}$ metodologia de modelos mistos, o ajuste de covariáveis é realizado simultaneamente à estimação e predição. Para isto foi utilizado o programa estatístico SAS (1999) por meio do procedimento Proc Mixed, utilizando o método REML.

O caráter volume foi avaliado quanto aos parâmetros genéticos e seus componentes de variância (herdabilidade, variância genotípica entre e dentro de famílias, erro experimental entre e dentro de parcelas), com e sem o emprego das covariáveis. $\mathrm{O}$ erro e o coeficiente de variação do EXP1 foram estimados com os dados das testemunhas (clones) gerando um erro puro, sem a presença da variância genotípica. Para oEXP2, a variação genotípica dentro de famílias foi estimada calculando a percentagem de cada componente de variância em relação ao total da variância do EXP1, e esta percentagem foi usada para calcular o erro dentro de parcelas no EXP2, e a variância genotípica dentro de famílias foi estimada subtraindo-se os demais componentes de variância $\left(\sigma_{e}^{2}\right.$ variância do erro experimental entre parcelas, $\sigma_{g e}{ }^{2}$ variância genotípica entre famílias e $\sigma_{e d}{ }^{2}$ variância do erro experimental dentro de parcelas) do total de variância observada no EXP2. Assim, no EXP2 o erro dentro de parcelas será da mesma magnitude em relação à variância total do EXP1.

\section{RESULTADOS E DISCUSSÃO}

As variações nos componentes de variância podem ser positivas ou negativas, conforme a covariável empregada. Quando esta for positiva na estimativa de componente de variância genotípica significa que a amplitude entre os materiais genéticos será maior, ou seja, aumentará a discrepância dos melhores materiais em relação aos piores. Já para os componentes de variância do erro dentro de parcelas $\left(\hat{\sigma}_{e d}^{2}\right)$ e entre parcelas $\left(\hat{\sigma}_{e}^{2}\right)$, a redução de ambos implicará numa melhoria ambiental proporcionando dados mais confiáveis para a seleção, onde estas variações ocorrem dentro da parcela, para $\hat{\sigma}_{e d}^{2}$, e entre as parcelas, para $\hat{\sigma}_{e}^{2}$, causando uma uniformidade ou desuniformidade entre as plantas da mesma parcela e entre os blocos, respectivamente. De maneira geral, os coeficientes da regressão linear das covariáveis apresentaram-se de forma ideal, exceto para o índice de competição (IC), nos dois experimentos, que apresentou interação tratamentos x covariável, e para a alocompetição (AL) no EXP1, que foi não significativa. O IC só pode ser usado após a remoção dos dados relativos às árvores de crescimento muito inferior (discrepantes) à média do experimento, em que, provavelmente, são indivíduos com alta probabilidade de morte. Assim, após a remoção dos dados destas árvores, 
esta covariável não apresentou mais interação significativa com os tratamentos.

As estimativas do coeficiente de variação experimental (CV) foram altas (Tabela 1), porque a característica analisada é composta por outras duas características, altura e diâmetro a altura do peito, como comprovado por outros autores (ANDRADE et al., 2006) que observaram CV acima de $30 \%$ para testes clonais.

A covariável AL não foi significativa no EXP1 e foi no EXP2, todavia, não influenciou na análise, individualmente ou em associação com outras covariáveis, não justificando a sua adoção na metodologia de análise para testes de progênies de eucalipto. Por isto, esses resultados não foram aqui apresentados.

TABELA 1: Componentes de variância, parâmetros genéticos, diferencial em porcentagem (\% de var.) e coeficiente de variação experimental $(\mathrm{CV})$ de dois testes de progênies de Eucalipto sem o uso (Usual) e com adoção de covariáveis de competição (Autocompetição-AT, Média da autocompetição-MAT, Alocompetição-AL, Média da alocompetição-MAL, Índice de competição-IC e Média 4 e 8 - M4, M8, respectivamente).

TABLE 1: Components of variance, genetic parameters, difference in percentage (\% of var.) and Experimental coefficient of variation (CV) of two tests of progenies of Eucalyptus without the use (Usual) and adoption of competition covariates (self-competition-AT , Mean selfcompetition -MAT, Alo competition-AL, Mean alo competition-MAL, competition index -IC and Mean 4 and 8 - M4, M8, respectively).

\begin{tabular}{|c|c|c|c|c|c|c|c|c|}
\hline \multirow{2}{*}{$\begin{array}{l}\text { Comp. de var. e } \\
\text { Par. Gen. } \\
\text { Exp1 }\end{array}$} & \multicolumn{7}{|c|}{ Métodos de Análise } & \multirow[b]{2}{*}{ M8 } \\
\hline & Usual & AT & MAT & $\mathrm{AL}$ & MAL & IC & M4 & \\
\hline$\hat{\sigma}_{g e}^{2}$ & 0,0038 & 0,0037 & 0,0051 & 0,0038 & 0,0036 & 0,0019 & 0,0047 & 0,0041 \\
\hline$\hat{\sigma}_{g d}^{g e}$ & 0,0043 & 0,0045 & 0,0046 & 0,0043 & 0,0042 & 0,0028 & 0,0046 & 0,0044 \\
\hline$\hat{\sigma}_{e}^{2 d}$ & 0,0012 & 0,0011 & 0,0018 & 0,0012 & 0,0010 & 0,0008 & 0,0014 & 0,0011 \\
\hline$\hat{\sigma}_{e d}^{e}$ & 0,0084 & 0,0081 & 0,0077 & 0,0084 & 0,0084 & 0,0047 & 0,0078 & 0,0080 \\
\hline$\%$ de var. $\hat{\sigma}_{g e}^{2}$ & - & $-1,86$ & 33,52 & $-0,18$ & $-4,84$ & $-50,62$ & 22,57 & 8,25 \\
\hline$\%$ de var. $\hat{\sigma}_{g d}^{2 e}$ & - & 6,75 & 9,19 & 0,16 & $-2,00$ & $-34,23$ & 7,03 & 2,68 \\
\hline$\%$ de var. $\hat{\sigma}_{e}^{2 a}$ & - & $-5,37$ & 50,63 & $-0,17$ & $-17,55$ & $-32,58$ & 15,03 & $-5,12$ \\
\hline$\%$ de var. $\hat{\sigma}_{e d}^{2}$ & - & $-3,99$ & $-8,13$ & $-0,12$ & 0,31 & $-44,05$ & $-7,85$ & $-4,72$ \\
\hline$\hat{h}_{e}^{2}$ & 28,44 & 28,93 & 34,86 & 28,43 & 27,83 & 25,49 & 33,91 & 31,12 \\
\hline$\hat{h}_{d}^{e}$ & 30,69 & 33,03 & 32,77 & 30,75 & 30,67 & 33,66 & 33,28 & 32,31 \\
\hline $\mathrm{CV} \%$ & 36,36 & 35,70 & 34,81 & 36,36 & 36,36 & 27,20 & 35,03 & 35,48 \\
\hline \multicolumn{9}{|l|}{ Exp2 } \\
\hline$\hat{\sigma}_{g e}{ }^{2}$ & 0,000833 & 0,000875 & 0,001462 & 0,000871 & 0,000753 & 0,000385 & 0,001265 & 0,001101 \\
\hline$\hat{\sigma}_{g d}^{g e}$ & 0,00853 & 0,00853 & 0,00862 & 0,00847 & 0,00812 & 0,00452 & 0,00859 & 0,00810 \\
\hline$\hat{\sigma}_{e}^{2}$ & 0 & 0 & 0,000878 & 0 & 0 & 0,000357 & 0,000375 & 0,000195 \\
\hline$\hat{\sigma}_{e d}{ }^{2}$ & 0,00844 & 0,008150 & 0,00748 & 0,00842 & 0,00844 & 0,00459 & 0,00760 & 0,00791 \\
\hline$\%$ de var, $\hat{\sigma}_{g e}^{2}$ & - & 5,04 & 75,51 & 4,56 & $-9,60$ & $-53,78$ & 51,86 & 32,17 \\
\hline$\%$ de var, $\hat{\sigma}_{g d}^{2 e}$ & - & $-0,02$ & 0,94 & $-0,71$ & $-4,81$ & $-46,99$ & 0,64 & $-5,15$ \\
\hline$\%$ de var, $\hat{\sigma}_{e}^{2}$ & - & - & - & - & - & - & - & - \\
\hline$\%$ de var, $\hat{\sigma}_{e d}^{2}$ & - & $-3,34$ & $-11,27$ & $-0,13$ & 0,07 & $-45,54$ & $-9,85$ & $-6,25$ \\
\hline$\hat{h}_{e}^{2}$ & 8,99 & 9,70 & 14,89 & 9,37 & 8,19 & 7,22 & 13,69 & 11,97 \\
\hline$\hat{h}_{d}^{e}$ & 50,30 & 51,15 & 50,75 & 50,15 & 49,05 & 47,75 & 51,85 & 49,98 \\
\hline $\begin{array}{l}n_{d} \\
\mathrm{CV} \%\end{array}$ & 39,58 & 38,91 & 37,28 & 39,55 & 39,59 & 29,21 & 37,58 & 38,32 \\
\hline
\end{tabular}

Em que: $\hat{\sigma}_{g e}{ }^{2}=$ variância genotípica entre famílias; $\hat{\sigma}_{g d}{ }^{2}=$ variância genotípica dentro de famílias; $\hat{\sigma}_{e}{ }^{2}=$ variância do erro experimental entre parcelas; $\hat{\sigma}_{e d}{ }^{2}=$ variância do erro experimental dentro de parcelas; $\hat{h}_{e}{ }^{2}=$ herdabilidade no sentido restrito entre famílias; $\hat{h}_{d}{ }^{2}=$ herdabilidade no sentido restrito dentro de famílias de meios-irmãos; CV\% $=$ coeficiente de variação experimental. 
A covariável de autocompetição (AT) proporcionou redução no erro dentro de parcelas, para ambos os experimentos e no erro entre parcelas para o EXP1, não influenciando este no EXP2. Contudo, para a variância genotípica entre tratamentos o comportamento foi distinto, ocasionando leve aumento no EXP2 e pequena redução no EXP1. Para a variância genotípica dentro de progênies houve aumento no EXP1 e não influenciou no EXP2. A herdabilidade apresentou pequeno aumento nos dois experimentos e o coeficiente de variação experimental (CV) sofreu pequena redução. Esta covariável não proporcionou uma correção homogênea, para efeito de falha dentro parcela, para os dois experimentos.

A média da autocompetição (MAT) proporcionou aumento na variabilidade genética entre famílias, porém, de forma mais expressiva no EXP2; e aumento no erro entre parcelas e redução no erro dentro de parcelas, resultando em maiores estimativas de herdabilidade entre famílias e menores $\mathrm{CV}$. Os resultados desta covariável sugerem que a autocompetição influenciou nos resultados, de forma que, quando as plantas de uma progênie estão sujeitas a autocompetição, as famílias se diferenciam menos umas das outras; também, a autocompetição reduz o $\hat{\sigma}_{e}^{2}$, sendo que, quando não há autocompetição nota-se uma diferenciação maior entre as parcelas de mesma família, ocasionando interação genótipo x bloco. Estes resultados corroboram com Scarpinati (2007), que notou o mesmo efeito da autocompetição, em testes clonais de eucalipto, em diferentes arranjos experimentais, pois arranjos experimentais com maior grau de autocompetição ocasionaram menores valores da variância genotípica e erro entre parcelas.

A média da alocompetição (MAL), também proporcionou resultados semelhantes em ambos os experimentos, causando redução generalizada nas variâncias, exceto na variação do erro dentro de parcelas. A variância que apresentou a maior queda foi $\hat{\sigma}_{e}^{2}$, no EXP1. Estes resultados sugerem que a competição entre materiais diferentes, nas parcelas adjacentes, acentua a magnitude genotípica dos materiais e também a diferença de um determinado genótipo em suas diferentes parcelas, conforme a agressividade competicional de seu vizinho. Logo, quando o efeito da competição entre os diferentes materiais é corrigido pela covariável de competição intergenotípica, as estimativas das variâncias genotípicas e do erro experimental tendem a diminuir. Outros autores também observaram o mesmo efeito da competição intergenotípica. Leornadecz-Neto et al. (2003), em teste de progênies de essências florestais, obtiveram reduções significativas nas variâncias, em diferentes graus, nas diferentes espécies estudadas. Lins (2004) observou o mesmo efeito em três espécies arbóreas nativas do Brasil. Já, Scarpinati (2007) notou que a variância genotípica aumentou conforme o arranjo experimental (tamanho da parcela experimental) propiciando uma maior competição intergenotípica, porém, esta sofreu redução, quando aplicada ao modelo matemático uma covariável de correção da competição.

O IC proporcionou as alterações mais pronunciadas nos dois experimentos, reduzindo de forma expressiva todos os componentes de variância. Isso indicou que grande parte da diferenciação dos materiais genéticos em testes de progênies é devida a competição entre e dentro de famílias. Esse mesmo efeito foi notado por Leornadecz-Neto et al. (2003) e por Lins (2004). Porém, esta covariável deve ser aplicada com cautela, corrigindo-se os dados originais pela eliminação das plantas de baixo rendimento. Scarpinati (2007), em testes clonais de eucalipto, não utilizou esta covariável, pois a competição que foi corrigida por este índice, pareceu ter efeito genético, em que os materiais mais produtivos geralmente também foram os mais competitivos, e a média experimental foi maior quando o nível de competição intergenotípica aumentou.

As médias dos quatro e dos oito vizinhos mais próximos (M4 e M8, respectivamente) proporcionaram resultados com a mesma tendência nos dois experimentos, em que M4 proporcionou variações mais expressivas nas estimativas de variâncias genotípicas e erros experimentais. Esta covariável proporcionou resultados semelhantes à MAT, o que pode ser explicado pelo fato destas terem formação parecida. Já, M8 parece ter mesclado os resultados obtidos com MAT e MAL, o que se deve ao fato de ser formada pelas duas anteriores.

Das análises de associação entre as covariáveis, são apresentados apenas os resultados mais promissores (Tabela 2), desconsiderando-se as combinações de covariáveis semelhantes em sua formação, MAL-M8 e MAT-M4, já que estas, quando utilizadas conjuntamente, resultaram na perda de significância de uma delas na análise. 
A covariável AT não proporcionou resultados satisfatórios quando em combinação com outras covariáveis, perdendo a significância e não alterando os resultados obtidos isoladamente. Disto, pode-se entender que o nível de falha na parcela influencia diferentemente os diversos experimentos e materiais genéticos, fato também observado por Andrade et al. (2006) em testes clonais de Eucalyptus urophylla, em que a resposta da compensação de falha na parcela foi dependente das condições edafoclimáticas e dos clones.

Por isso, e pela não significância $(p>0,05)$ da covariável AL no EXP1, a ausência de competição parece proporcionar respostas diferentes para cada experimento, de forma que os materiais genéticos compensam ou não a falha de plantas adjacentes,

TABELA 2: Componentes de variância, parâmetros genéticos, diferencial em porcentagem (\% de var,) e coeficiente de variação experimental $(\mathrm{CV})$ de dois experimentos de testes de progênies de eucalipto sem o uso (Usual) e com adoção de associações de covariáveis de correção de competição (Média da autocompetição-MAT, Média da alocompetição-MAL, Índice de competição-IC e Média 4 e 8 -M4, M8), respectivamente.

TABLE 2: Components of variance, genetic parameters, difference in percentage ( $\%$ of variation, ) and experimental coefficient of variation $(\mathrm{CV})$ of two progenies of Eucalyptus test without the use (Usual) and adoption by associations of covariates for correction of competition (MATAverage self-competition, Average alo competition-MAL, competition index IC and average -4 and 8-M4, M8), respectively.

\begin{tabular}{|c|c|c|c|c|c|c|c|c|c|}
\hline $\begin{array}{l}\text { Comp, de var, } \\
\text { e Par, Gen, }\end{array}$ & & & & Mét & dos de Anál & & & & \\
\hline Exp1 & Usual & MAT/MAL & MAT/IC & MAL/IC & MAL/M4 & IC/M4 & IC/M8 & $\begin{array}{c}\text { MAT } \\
\text { MAL/IC }\end{array}$ & $\begin{array}{c}\text { MAL/IC } \\
\text { M4 }\end{array}$ \\
\hline$\hat{\sigma}_{g e}^{2}$ & 0,0038 & 0,0049 & 0,0020 & 0,0020 & 0,0046 & 0,0019 & 0,0017 & 0,0020 & 0,0020 \\
\hline$\hat{\sigma}_{g d}^{2}$ & 0,0043 & 0,0045 & 0,0028 & 0,0027 & 0,0045 & 0,0028 & 0,0027 & 0,0027 & 0,0028 \\
\hline$\hat{\sigma}_{e}^{2}$ & 0,0012 & 0,0015 & 0,0008 & 0,0010 & 0,0013 & 0,0008 & 0,0009 & 0,0010 & 0,0010 \\
\hline$\hat{\sigma}_{e d}^{2}$ & 0,0084 & 0,0077 & 0,0047 & 0,0047 & 0,0078 & 0,0047 & 0,0048 & 0,0047 & 0,0046 \\
\hline$\%$ de var, $\hat{\sigma}_{g e}^{2}$ & - & 31,47 & $-47,63$ & $-48,49$ & 19,72 & $-51,19$ & $-54,65$ & $-47,63$ & $-46,35$ \\
\hline$\%$ de var, $\hat{\sigma}_{g d}^{g e}$ & - & 29,12 & $-29,81$ & $-17,30$ & 9,49 & $-32,91$ & $-26,95$ & $-16,46$ & $-15,87$ \\
\hline$\%$ de var, $\hat{\sigma}_{e}^{2}$ & - & 28,80 & $-33,36$ & $-35,74$ & 6,14 & $-34,59$ & $-37,50$ & $-35,46$ & $-34,73$ \\
\hline$\%$ de var, $\hat{\sigma}_{e d}^{2}$ & - & $-8,08$ & $-44,55$ & $-44,43$ & $-7,40$ & $-43,85$ & $-43,08$ & $-44,57$ & $-44,96$ \\
\hline$\hat{h}_{e}^{2}$ & 28,44 & 34,72 & 26,66 & 25,78 & 33,45 & 25,23 & 23,43 & 26,11 & 26,67 \\
\hline$\hat{h}_{d}^{2}$ & 30,69 & 32,90 & 33,99 & 32,56 & 33,16 & 33,49 & 31,95 & 32,66 & 33,01 \\
\hline $\mathrm{CV} \%$ & 36,36 & 34,81 & 27,20 & 27,20 & 35,03 & 27,20 & 27,48 & 27,20 & 26,90 \\
\hline Exp2 & & & & & & & & & \\
\hline$\hat{\sigma}_{g e}^{2}$ & 0,00083 & 0,00143 & 0,00049 & 0,00039 & 0,00119 & 0,00045 & 0,00041 & 0,00049 & 0,00045 \\
\hline$\hat{\sigma}_{g d}^{2 c}$ & 0,00852 & 0,00789 & 0,00416 & 0,00441 & 0,00819 & 0,00420 & 0,00415 & 0,00422 & 0,00437 \\
\hline$\hat{\sigma}_{e}^{2}$ & 0 & 0,00063 & 0,00049 & 0,00036 & 0,00024 & 0,00042 & 0,00037 & 0,00047 & 0,00043 \\
\hline$\hat{\sigma}_{e d}{ }^{2}$ & 0,00844 & 0,00703 & 0,00432 & 0,00425 & 0,00721 & 0,00434 & 0,00447 & 0,00427 & 0,00417 \\
\hline$\%$ de var, $\hat{\sigma}_{g e}^{2}$ & - & 71,67 & $-41,18$ & $-53,18$ & 42,86 & $-45,98$ & $-50,78$ & $-41,18$ & $-45,98$ \\
\hline$\%$ de var, $\hat{\sigma}_{g d}^{2}$ & - & $-7,34$ & $-51,11$ & $-48,24$ & $-3,86$ & $-50,63$ & $-51,24$ & $-50,45$ & $-48,64$ \\
\hline$\%$ de var, $\hat{\sigma}_{e}^{2}$ & - & - & - & - & - & - & - & - & - \\
\hline$\%$ de var, $\hat{\sigma}_{e d}^{2}$ & - & $-16,75$ & $-48,88$ & $-49,64$ & $-14,58$ & $-48,65$ & $-47,10$ & $-49,42$ & $-50,66$ \\
\hline$\hat{h}_{e}{ }^{2}$ & 8,98 & 15,73 & 9,25 & 7,80 & 13,77 & 8,64 & 7,81 & 9,37 & 8,92 \\
\hline$\hat{h}_{d}^{e}$ & 50,21 & 50,74 & 46,41 & 48,86 & 52,35 & 46,92 & 46,19 & 47,09 & 48,76 \\
\hline $\mathrm{CV} \%$ & 39,61 & 36,14 & 28,32 & 28,11 & 36,61 & 28,38 & 28,81 & 28,17 & 27,82 \\
\hline
\end{tabular}

Em que: $\hat{\sigma}_{g e}{ }^{2}=$ variância genotípica entre famílias; $\hat{\sigma}_{g d}{ }^{2}=$ variância genotípica dentro de famílias; $\hat{\sigma}_{e}{ }^{2}=$ variância do erro experimental entre parcelas; $\hat{\sigma}_{e d}{ }^{2}=$ variância do erro experimental dentro de parcelas; $\hat{h}_{e}{ }^{2}=$ herdabilidade no sentido restrito entre famílias; ${ }^{2}=$ herdabilidade no sentido restrito dentro de famílias de meios-irmãos; $\mathrm{CV} \%=$ coeficiente de variação expêtimental. 
sendo esta, na mesma parcela ou em parcelas vizinhas. Outrossim, além da resposta diferenciada às condições edafoclimáticas, os materiais genéticos podem ter aptidões diferenciadas, com alguns apresentando, ou não, aumento no crescimento, pela ausência de competição causada pela falha de plantas vizinhas. Esta aptidão ainda pode se manifestar apenas quando as condições ambientais forem propícias, conforme sugerido pelo fato de AL ter sido não significativa no EXP1, onde a média geral do experimento foi bem inferior à média do EXP2, de forma que, quando as condições ambientais não são favoráveis os genótipos não manifestam a sua capacidade para a compensação da falha.

Assim, pode-se considerar que esta característica pode ter origem genética, resultando em interações genótipo-ambiente, em que os materiais genéticos respondem de forma diferenciada à abertura de espaçamento, conforme as condições ambientais no experimento. Desta forma, todas as associações destas duas covariáveis (AL e AT) não foram aqui apresentadas.

As associações apresentadas proporcionaram resultados semelhantes nos dois experimentos. Contudo, para algumas associações, a variância genotípica dentro de famílias no EXP2 apresentou comportamento inverso ao EXP1, o que se deve ao fato desse parâmetro ter sido obtido a partir do erro dentro de parcela do EXP1, e quando este sofreu variações de magnitudes diferentes dos outros parâmetros, isso se refletiu na estimativa da variância genotípica dentro de famílias do EXP2. Desconsiderando este fato, a associação que proporcionou maior aumento nos parâmetros genéticos foi MAT/MAL como, por exemplo, na herdabilidade, além de ter reduzido o erro dentro de parcela. Outra associação promissora foi a MAL/M4, que também ocasionou aumento da herdabilidade e redução no $\mathrm{CV}$, porém, de forma menos pronunciada do que a associação anterior.

As associações do IC em relação à análise usual, de forma geral, ocasionaram quedas no $\mathrm{CV}$, nos parâmetros genéticos e no erro dentro de parcelas, porém, de maneira menos pronunciada para os parâmetros genéticos do que quando o IC foi aplicado isoladamente, principalmente para variação genética entre famílias.

Dentre as formas de competição, inter e intragenotípica, a intergenotípica é a mais importante para testes genéticos, já que esta faz com que a amplitude entre os materiais aumente assim como o erro entre parcelas. Essa é uma importante contribuição para futuros testes genéticos na seleção de genótipos superiores, pois, como a competição influencia no desempenho dos materiais de maneira diferenciada entre os mesmos, isso pode acarretar na supressão de alguns genótipos que poderiam ser promissores, ou ainda, na supervalorização de materiais altamente competitivos.

A competição intragenotípica não influencia tanto o desempenho dos materiais de mesma família, apesar de ser um teste de famílias de polinização aberta em que o parentesco fica condicionado somente à mãe, os materiais de mesma família não competem de forma tão intensa entre si, ou como apresentam potencial competitivo semelhante os efeitos causados pela alocompetição, como maior variância ambiental e genotípica, não são notados na autocompetição. Ainda, considerando que a competição intergenotípica pode ser maior pelo número de competidores, já que, a planta em questão, pode estar competindo com até sete árvores das parcelas vizinhas e no máximo duas dentro da mesma parcela, fazendo com que a planta objeto do estudo seja suprimida ou supressora de três árvores de cada lado das parcelas vizinhas, potencializando a competição intergenotípica em conjunto da parcela adjacente.

Uma das formas de se minimizar ou anular o efeito da competição intergenotípica seria adotar parcelas maiores, com formato retangular, com grande número de plantas em que seriam avaliadas somente as árvores no interior da parcela. Porém, com a necessidade de se testar um grande número de materiais genéticos e pelas características das árvores, demandaria grandes áreas, o que aumentaria os custos e dificultaria a experimentação pela heterogeneidade do ambiente. Logo, a adoção de métodos estatísticos que possibilitem a redução deste efeito se justifica.

Dentre as diversas formas de se avaliar o efeito da competição em testes genéticos se destaca a análise de covariável. Contudo, saber qual covariável e como esta se ajusta de forma a representar, com fidelidade, o efeito biológico, é uma incógnita. No presente trabalho constatou-se que a competição intergenotípica influencia nos resultados e as covariáveis que proporcionaram resultados mais promissores, foram aquelas que em sua formação consideraram as árvores das parcelas vizinhas, sendo elas a Média da Alocompetição (MAL) e o Índice de Competição de Hegyi (IC). Porém, estas covariáveis usadas em conjunto não proporcionaram resultados promissores, pois uma covariável se sobrepõe a outra, fazendo ajustes dos dados duas vezes para o mesmo efeito. 
Quando associados os efeitos da alo com os da autocompetição, adotando-se na mesma análise as covariáveis que os representam, obtevese resultados promissores com o uso simultâneo das covariáveis IC e da Média da Autocompetição (MAT), de forma que o IC interfere expressivamente na análise e a MAT interfere de forma oposta, equilibrando o efeito muito severo de IC. Assim, a combinação MAT/IC pode ser considerada a mais promissora por reduzir consideravelmente o erro dentro de parcela. Apesar de diminuir a variância genotípica, esta redução foi menor do que quando usado o IC isoladamente, resultando em ganho na herdabilidade. Este fato foi mais relevante no EXP2, em que a herdabilidade entre famílias foi menor.

\section{CONCLUSÕES}

As covariáveis mais promissoras para estudo de competição em testes de progênies de polinização aberta de eucalipto são: a média da autocompetição (MAT), média da alocompetição (MAL) e índice de competição de HegyI (IC);

A covariável autocompetição (AT) não proporcionou resultados conclusivos, parecendo ser dependente das condições genéticas e ambientais e, a covariável alocompetição (AL) não interfere na análise;

A autocompetição parece causar uma menor variabilidade genética e erro entre parcelas, já a alocompetição interfere de forma oposta em testes de progênies de eucalipto;

A rotina de análise que proporcionou melhores resultados foi a que incluiu as covariáveis MAT/IC, sendo promissora na redução do efeito competicional em testes de progênies de eucalipto.

\section{REFERÊNCIAS BIBLIOGRÁFICAS}

ANDRADE, H. B. et al. Alternativas para atenuar a diferença de estande nos experimentos de avaliação de clones de Eucalyptus urophylla. Revista Árvore, Viçosa, v. 30, n. 1, p.11-18, 2006. COSTA, R. B. da. et al. Prediction of genotypic values for yield in rubber tree-clone test trials using REML/BLUP procedure. Crop Breeding and Applied Biotechnology. Viçosa. v. 2, n. 4, p. 579-586, 2002.

DUARTE, J. B.; VENCOVSKY. R. Estimação e predição por modelo linear misto com ênfase na ordenação de médias de tratamentos genéticos.
Scientia Agricola, v. 58, n. 1, p. 109-117, jan/mar. 2001.

FALCONER, D. S. Introdução à genética quantitativa. Viçosa, UFV, 1987. 279 p.

GARCIA, C. H.; NOGUEIRA, M. C. S. N. Utilização da metodologia REML/BLUP na seleção de clones de eucalipto. Scientia Forestalis, Piracicaba, n. 68, p.107-112, 2005.

HEGYI, F. A simulation model for managing jack pine stands. In FRIES, J. (Ed) Growth models for tree and stand simulation. Stockholm: Department forest yield reserarch. 1974. p 74-85. LEONARDECZ-NETO, E.; VENCOVSKY, R.; SEBBENN, A. M. Ajuste para a competição entre plantas em teste de progênies e procedências de essências florestais. Scientia Forestalis, Piracicaba, n. 63, p. 136-149, jun. 2003.

LINS, V. S. Variação genética e competição intergenotípica em testes de progênie de Myracrodruon urundeuva Fr. All., Terminalia argentea Mart. Et Succ. e Astronium fraxinifolium Schott em consórcio. Ilha Solteira, 2004. 73 f. Dissertação (Mestrado em Agronomia), Universidade Estadual Paulista, Ilha Solteira, 2004.

RAMALHO, M. A. P.; FERREIRA, D. F.; OLIVEIRA, A. C. Experimentação em genética e melhoramento de plantas. Lavras: UFLA, 2000. $326 \mathrm{p}$.

RESENDE, M. D. V. Análise estatística de modelos mistos via REML/BLUP na experimentação em melhoramento de plantas perenes. Colombo: EMBRAPA Florestas, 2000. 101p. (EMBRAPA Florestas. documentos 47).

ROCHA, M. das G. de B. et al. Seleção de genitores de Eucalyptus grandis e de Eucalyptus urophylla para produção de híbridos interespecíficos utilizando REML/BLUP e informação de divergência genética. Revista Árvore. v. 31, n. 6, p. 977-987, 2007.

SAS. Institute inc. SAS procedures guide. Version 8 (TSMO). Cary: SAS institute Inc., 1999. 454 p. SCARPINATI, E. A. Influência do modelo de análise estatística e da forma das parcelas experimentais na seleção de clones de Eucalyptus spp. Jaboticabal, 2007. 58f. Dissertação (mestrado em Agronomia)-Universidade Estadual Paulista, Jaboticabal, 2007.

SIMEÃO, R. M. et al. Avaliação genética em erva-mate pelo procedimento BLUP individual multivariado sob interação genótipo $\mathrm{x}$ ambiente. Pesquisa Agropecuária Brasileira, Brasília. v. 
37, n. 11, p. 1589-1596, 2002.

STORCK, L.; VENCOVSKY, R. Stability analysis based on a bi-segmented discontinuous model with measurement erros in the variables. . Brazilian Journal of Genetics, Ribeirão Preto, v. 17, n. 1, p. 75-81, 1994. 